\title{
Another Generalization of Abelian Equivalence: Binomial Complexity of Infinite Words
}

\author{
M. Rigo ${ }^{1}$ and P. Salimov ${ }^{1,2 \star}$ \\ 1 Dept of Math., University of Liège, Grande traverse 12 (B37), B-4000 Liège, \\ Belgium, M.Rigo@ulg.ac.be \\ 2 Sobolev Institute of Math., 4 Acad. Koptyug avenue, 630090 Novosibirsk, Russia.
}

\begin{abstract}
The binomial coefficient of two words $u$ and $v$ is the number of times $v$ occurs as a subsequence of $u$. Based on this classical notion, we introduce the $m$-binomial equivalence of two words refining the abelian equivalence. The $m$-binomial complexity of an infinite word $x$ maps an integer $n$ to the number of $m$-binomial equivalence classes of factors of length $n$ occurring in $x$. We study the first properties of $m$-binomial equivalence. We compute the $m$-binomial complexity of the Sturmian words and of the Thue-Morse word. We also mention the possible avoidance of 2-binomial squares.
\end{abstract}

\section{Introduction}

In the literature, many measures of complexity of infinite words have been introduced. One of the most studied is the factor complexity $p_{x}$ counting the number of distinct blocks of $n$ consecutive letters occurring in an infinite word $x \in A^{\mathbb{N}}$. In particular, Morse-Hedlund theorem gives a characterization of ultimately periodic words in terms of bounded factor complexity. Sturmian words have a null topological entropy and are characterized by the relation $p_{x}(n)=n+1$ for all $n \geqslant 0$. Abelian complexity counts the number of distinct Parikh vectors for blocks of $n$ consecutive letters occurring in an infinite word, i.e., factors of length $n$ are counted up to abelian equivalence. Already in 1961, Erdős opened the way to a new research direction by raising the question of avoiding abelian squares in arbitrarily long words [6]. Related to Van der Waerden theorem, we can also mention the arithmetic complexity [1] mapping $n \geqslant 0$ to the number of distinct subwords $x_{i} x_{i+p} \cdots x_{i+(n-1) p}$ built from $n$ letters arranged in arithmetic progressions in the infinite word $x, i \geqslant 0, p \geqslant 1$. In the same direction, one can also consider maximal pattern complexity [7].

As a generalization of abelian complexity, the $k$-abelian complexity was recently introduced through a hierarchy of equivalence relations, the coarsest being abelian equivalence and refining up to equality. We recall these notions.

\footnotetext{
^ The second author is supported by the Russian President's grant no. MK-4075.2012.1 and Russian Foundation for Basic Research grants no. 12-01-00089 and no. 11-0100997 and by a University of Liège post-doctoral grant.
} 
Let $k \in \mathbb{N} \cup\{+\infty\}$ and $A$ be a finite alphabet. As usual, $|u|$ denotes the length of $u$ and $|u|_{x}$ denotes the number of occurrences of the word $x$ as a factor of the word $u$. Karhumäki et al. [8] introduce the notion of $k$-abelian equivalence of finite words as follows. Let $u, v$ be two words over $A$. We write $u \sim_{\text {ab, } k} v$ if and only if $|u|_{x}=|v|_{x}$ for all words $x$ of length $|x| \leqslant k$. In particular, $u \sim_{\text {ab, } 1} v$ means that $u$ and $v$ are abelian equivalent, i.e., $u$ is obtained by permuting the letters in $v$.

The aim of this paper is to introduce and study the first properties of a different family of equivalence relations over $A^{*}$, called $k$-binomial equivalence, where the coarsest relation coincide with the abelian equivalence.

Let $u=u_{0} \cdots u_{n-1}$ be a word of length $n$ over $A$. Let $\ell \leqslant n$. Let $t: \mathbb{N} \rightarrow \mathbb{N}$ be an increasing map such that $t(\ell-1)<n$. Then the word $u_{t(0)} \cdots u_{t(\ell-1)}$ is a subword of length $\ell$ of $u$. Note that what we call subword is also called scattered subword in the literature. The notion of binomial coefficient of two finite words $u$ and $v$ is well-known, $\left(\begin{array}{l}u \\ v\end{array}\right)$ is defined as the number of times $v$ occurs as a subword of $u$. In other words, the binomial coefficient of $u$ and $v$ is the number of times $v$ appears as a subsequence of $u$. Properties of these coefficients are presented in the chapter of Lothaire's book written by Sakarovitch and Simon [12, Section $6.3]$. Let $a, b \in A, u, v \in A^{*}$ and $p, q$ be integers. We set $\delta_{a, b}=1$ if $a=b$, and $\delta_{a, b}=0$ otherwise. We just recall that

$$
\left(\begin{array}{l}
a^{p} \\
a^{q}
\end{array}\right)=\left(\begin{array}{l}
p \\
q
\end{array}\right),\left(\begin{array}{l}
u \\
\varepsilon
\end{array}\right)=1,|u|<|v| \Rightarrow\left(\begin{array}{l}
u \\
v
\end{array}\right)=0,\left(\begin{array}{c}
u a \\
v b
\end{array}\right)=\left(\begin{array}{c}
u \\
v b
\end{array}\right)+\delta_{a, b}\left(\begin{array}{l}
u \\
v
\end{array}\right)
$$

and the last three relations completely determine the binomial coefficient $\left(\begin{array}{l}u \\ v\end{array}\right)$ for all $u, v \in A^{*}$.

Remark 1 . Note that we have to make a distinction between subwords and factors. A factor is a particular subword made of consecutive letters. Factors of $u$ are denoted either by $u_{i} \cdots u_{j}$ or $u[i, j], 0 \leqslant i \leqslant j<|u|$.

Definition 1. Let $m \in \mathbb{N} \cup\{+\infty\}$ and $u, v$ be two words over $A$. We say that $u$ and $v$ are $m$-binomially equivalent if

$$
\left(\begin{array}{l}
u \\
x
\end{array}\right)=\left(\begin{array}{l}
v \\
x
\end{array}\right), \forall x \in A^{\leqslant m} .
$$

Since the main relation studied in this paper is the $m$-binomial equivalence, we simply write in that case: $u \sim_{m} v$.

Since $\left(\begin{array}{c}u \\ a\end{array}\right)=|u|_{a}$ for all $a \in A$, it is clear that two words $u$ and $v$ are abelian equivalent if and only if $u \sim_{1} v$. As for abelian equivalence, we have a family of refined relations: for all $u, v \in A^{*}, m \geqslant 0, u \sim_{m+1} v \Rightarrow u \sim_{m} v$.

Example 1. For instance, the four words $a b a b b b a, a b b a b a b, b a a b b a b$ and $b a b a a b b$ are 2-binomially equivalent. For any $w$ amongst these words, we have the following coefficients

$$
\left(\begin{array}{l}
w \\
a
\end{array}\right)=3,\left(\begin{array}{c}
w \\
b
\end{array}\right)=4,\left(\begin{array}{c}
w \\
a a
\end{array}\right)=3,\left(\begin{array}{c}
w \\
a b
\end{array}\right)=7,\left(\begin{array}{c}
w \\
b a
\end{array}\right)=5,\left(\begin{array}{c}
w \\
b b
\end{array}\right)=6 .
$$


But one can check that they are not 3-binomially equivalent, as an example,

$$
\left(\begin{array}{c}
a b a b b b a \\
a a b
\end{array}\right)=3 \text { but }\left(\begin{array}{c}
a b b a b a b \\
a a b
\end{array}\right)=4
$$

indeed, for this last binomial coefficient, $a a b$ appears as subwords $w_{0} w_{3} w_{4}$, $w_{0} w_{3} w_{6}, w_{0} w_{5} w_{6}$ and $w_{3} w_{5} w_{6}$. Considering again the first two words, we find $|a b a b b b a|_{a b}=2$ and $|a b b a b a b|_{a b}=3$, showing that these two words are not 2abelian equivalent. Conversely, the words abbaba and $a b a b b a$ are 2-abelian equivalent but are not 2-binomially equivalent:

$$
\left(\begin{array}{c}
a b b a b a \\
a b
\end{array}\right)=4 \text { but }\left(\begin{array}{c}
a b a b b a \\
a b
\end{array}\right)=5 \text {. }
$$

This paper is organized as follows. In the next section, we present some straightforward properties of binomial coefficients and $m$-binomial equivalence. In Section 3, we give upper bounds on the number of $m$-binomial equivalence classes partitioning $A^{n}$. Section 3 ends with the introduction of the $m$-binomial complexity $\mathbf{b}_{x}^{(m)}$ of an infinite word $x$. In Section 4 , we prove that if $x$ is a Sturmian word then, for any $m \geqslant 2, \mathbf{b}_{x}^{(m)}(n)=n+1$ for all $n \geqslant 0$. In Section 5 we consider the Thue-Morse word $t$ and show that, for all $m \geqslant 1$, there exists a constant $C_{m}$ such that $\mathbf{b}_{t}^{(m)}(n) \leqslant C_{m}$ for all $n \geqslant 0$. For instance, binomial coefficients of $t$ were considered in [3]. Due to space limitations, we only give details for the cases $m=2,3$. In the last section, we evoke the problem of avoiding 2-binomial squares.

\section{First Properties}

We denote by $\mathbf{B}^{(m)}(v)$ the equivalence class of words $m$-binomially equivalent to $v$. Binomial coefficients have a nice behavior with respect to the concatenation of words.

Proposition 1. Let $p, s$ and $e=e_{0} e_{1} \cdots e_{n-1}$ be finite words. We have

$$
\left(\begin{array}{c}
p s \\
e
\end{array}\right)=\sum_{i=0}^{n}\left(\begin{array}{c}
p \\
e_{0} e_{1} \cdots e_{i-1}
\end{array}\right)\left(\begin{array}{c}
s \\
e_{i} e_{i+1} \cdots e_{n-1}
\end{array}\right) \text {. }
$$

We can also mention some other basic facts on $m$-binomial equivalence.

Lemma 1. Let $u, u^{\prime}, v, v^{\prime}$ be finite words and $m \geqslant 1$.

- If $u \sim_{m} v$, then $u \sim_{\ell} v$ for all $\ell \leqslant m$.

- If $u \sim_{m} v$ and $u^{\prime} \sim_{m} v^{\prime}$, then $u u^{\prime} \sim_{m} v v^{\prime}$.

Proof. Simply note for the second point that, for all $x=x_{0} \cdots x_{\ell-1}$ of length $\ell \leqslant m,\left(\begin{array}{c}u u^{\prime} \\ x\end{array}\right)$ is equal to

$$
\sum_{i=0}^{\ell}\left(\begin{array}{c}
u \\
x[0, i-1]
\end{array}\right)\left(\begin{array}{c}
u^{\prime} \\
x[i, \ell-1]
\end{array}\right)=\sum_{i=0}^{\ell}\left(\begin{array}{c}
v \\
x[0, i-1]
\end{array}\right)\left(\begin{array}{c}
v^{\prime} \\
x[i, \ell-1]
\end{array}\right)=\left(\begin{array}{c}
v v^{\prime} \\
x
\end{array}\right) .
$$


Remark 2. Thanks to the above lemma, we can endow the quotient set $A^{*} / \sim_{m}$ with a monoid structure using an operation $\circ: A^{*} / \sim_{m} \times A^{*} / \sim_{m} \rightarrow A^{*} / \sim_{m}$ defined by $\mathbf{B}^{(m)}(p) \circ \mathbf{B}^{(m)}(q)=\mathbf{B}^{(m)}(r)$ if the concatenation $\mathbf{B}^{(m)}(p) . \mathbf{B}^{(m)}(q)$ is a subset of $\mathbf{B}^{(m)}(r)$. In particular, one can take $r=p q$. If a word $v$ is factorized as $v=p u s$, then the $m$-equivalence class $\mathbf{B}^{(m)}(v)$ is completely determined by $p, s$ and $\mathbf{B}^{(m)}(u)$.

\section{On the Number of $k$-Binomial Equivalence Classes}

For 2- and 3-abelian equivalence, the number of equivalence classes for words of length $n$ over a binary alphabet are respectively $n^{2}-n+2$ and $\Theta\left(n^{4}\right)$. In general, for $k$-abelian equivalence, the number of equivalence classes for words of length $n$ over a $\ell$-letter alphabet is $\Theta\left(n^{(\ell-1) \ell^{k-1}}\right)$ [8]. We consider similar results for $m$-binomial equivalence (proofs can be found in [15]).

Lemma 2. Let $u \in A^{*}, a \in A$ and $\ell \geqslant 0$. We have

$$
\left(\begin{array}{c}
u \\
a^{\ell}
\end{array}\right)=\left(\begin{array}{c}
|u|_{a} \\
\ell
\end{array}\right) \quad \text { and } \quad \sum_{|v|=\ell}\left(\begin{array}{l}
u \\
v
\end{array}\right)=\left(\begin{array}{c}
|u| \\
\ell
\end{array}\right) .
$$

Lemma 3. Let $A$ be a binary alphabet, we have

$$
\#\left(A^{n} / \sim_{2}\right)=\sum_{j=0}^{n}((n-j) j+1)=\frac{n^{3}+5 n+6}{6} .
$$

Proposition 2. Let $m \geqslant 2$. Let $A$ be a binary alphabet, we have

$$
\#\left(A^{n} / \sim_{m}\right) \in \mathcal{O}\left(n^{2\left((m-1) 2^{m}+1\right)}\right) .
$$

We denote $\operatorname{by~} \operatorname{Fac}_{x}(n)$ the set of factors of length $n$ occurring in $x$.

Definition 2. Let $m \geqslant 1$. The $m$-binomial complexity of an infinite word $x$ counts the number of $m$-binomial equivalence classes of factors of length $n$ occurring in $x$,

$$
\mathbf{b}_{x}^{(m)}: \mathbb{N} \rightarrow \mathbb{N}, n \mapsto \#\left(\operatorname{Fac}_{x}(n) / \sim_{m}\right) .
$$

Note that $\mathbf{b}_{x}^{(1)}$ corresponds to the usual abelian complexity denoted by $\rho_{x}^{a b}$.

If $p_{x}$ denotes the usual factor complexity, then for all $m \geqslant 1$, we have

$$
\mathbf{b}_{x}^{(m)}(n) \leqslant \mathbf{b}_{x}^{(m+1)}(n) \quad \text { and } \quad \rho_{x}^{\mathrm{ab}}(n) \leqslant \mathbf{b}_{x}^{(m)}(n) \leqslant p_{x}(n) .
$$

\section{The $m$-Binomial Complexity of Sturmian Words}

Recall that a Sturmian word $x$ is a non-periodic word of minimal (factor) complexity, that is, $p_{x}(n)=n+1$ for all $n \geqslant 0$. The following characterization is also useful. 
Theorem 1. [13, Theorem 2.1.5] An infinite word $x \in\{0,1\}^{\omega}$ is Sturmian if and only if it is aperiodic and balanced, i.e., for all factors $u, v$ of the same length occurring in $x$, we have $\left.|| u\right|_{1}-|v|_{1} \mid \leqslant 1$.

The aim of this section is to compute the $m$-binomial complexity of a Sturmian word as expressed by Theorem 2. We show that any two distinct factors of length $n$ occurring in a Sturmian words are never $m$-binomially equivalent. First note that Sturmian words have a constant abelian complexity. Hence, if $x$ is a Sturmian word, then $\mathbf{b}_{x}^{(1)}(n)=2$ for all $n \geqslant 1$.

Theorem 2. Let $m \geqslant 2$. If $x$ is a Sturmian word, then $\mathbf{b}_{x}^{(m)}(n)=n+1$ for all $n \geqslant 0$.

Remark 3. If $x$ is a right-infinite word such that $\mathbf{b}_{x}^{(1)}(n)=2$ for all $n \geqslant 1$, then $x$ is clearly balanced. If $\mathbf{b}_{x}^{(2)}(n)=n+1$, for all $n \geqslant 0$, then the factor complexity function $p_{x}$ is unbounded and $x$ is aperiodic. As a consequence of Theorem 2 , an infinite word $x$ is Sturmian if and only if, for all $n \geqslant 1$ and all $m \geqslant 2, \mathbf{b}_{x}^{(1)}(n)=2$ and $\mathbf{b}_{x}^{(m)}(n)=n+1$.

Before proceeding to the proof of Theorem 2, we first recall some well-known fact about Sturmian words. One of the two symbols occurring in a Sturmian word $x$ over $\{0,1\}$ is always isolated, for instance, 1 is always followed by 0 . In that latter case, there exists a unique $k \geqslant 1$ such that each occurrence of 1 is always followed by either $0^{k} 1$ or $0^{k+1} 1$ and $x$ is said to be of type 0 . See for instance [14, Chapter 6]. More precisely, we have the following remarkable fact showing that the recoding of a Sturmian sequence corresponds to another Sturmian sequence. Note that $\sigma: A^{\omega} \rightarrow A^{\omega}$ is the shift operator mapping $\left(x_{n}\right)_{n \geqslant 0}$ to $\left(x_{n+1}\right)_{n \geqslant 0}$.

Theorem 3. Let $x \in\{0,1\}^{\omega}$ be a Sturmian word of type 0. There exists a unique integer $k \geqslant 1$ and a Sturmian word $y \in\{0,1\}^{\omega}$ such that $x=\sigma^{c}(\mu(y))$ for some $c \leqslant k+1$ and where the morphism $\mu:\{0,1\}^{*} \rightarrow\{0,1\}^{*}$ is defined by $\mu(0)=0^{k} 1$ and $\mu(1)=0^{k+1} 1$.

Corollary 1. Let $x \in\{0,1\}^{\omega}$ be a Sturmian word of type 0. There exists a unique integer $k \geqslant 1$ such that any factor occurring in $x$ is of the form

$$
0^{r} 10^{k+\epsilon_{0}} 10^{k+\epsilon_{1}} 1 \cdots 0^{k+\epsilon_{n-1}} 10^{s}
$$

where $r, s \leqslant k+1$ and $\epsilon_{0} \epsilon_{1} \cdots \epsilon_{n-1} \in\{0,1\}^{*}$ is a factor of the Sturmian word $y$ introduced in the above theorem.

Let $\epsilon=\epsilon_{0} \cdots \epsilon_{n-1}$ be a word over $\{0,1\}$. For $m \leqslant n-1$, we define

$$
S(\epsilon, m):=\sum_{j=0}^{m}(n-j) \epsilon_{j} \quad \text { and } \quad S(\epsilon):=S(\epsilon, n-1) .
$$


Remark 4. Let $v=0^{r} 10^{k+\epsilon_{0}} 10^{k+\epsilon_{1}} 1 \cdots 0^{k+\epsilon_{n-1}} 10^{s}$ of the form (2), we have

$$
\left(\begin{array}{c}
v \\
01
\end{array}\right)=r(n+1)+\sum_{j=0}^{n-1}\left(k+\epsilon_{j}\right)(n-j)=r(n+1)+S\left(\epsilon_{0} \cdots \epsilon_{n-1}\right)+k \frac{n(n+1)}{2} .
$$

We need a technical lemma on the factors of a Sturmian word.

Lemma 4. Let $n \geqslant 1$. If $u$ and $v$ are two distinct factors of length $n$ occurring in a Sturmian word over $\{0,1\}$, then $S(u) \not \equiv S(v)(\bmod n+1)$.

Proof. Consider two distinct factors $u, v$ of length $n$ occurring in a Sturmian word $y$. For $m<n$, we define $\Delta(m):=\left|u_{0} u_{1} \cdots u_{m}\right|_{1}-\left|v_{0} v_{1} \cdots v_{m}\right|_{1}$. Due to Theorem 3, we have $|\Delta(m)| \leqslant 1$. Note that, if there exists $i$ such that $\Delta(i)=1$ then, for all $j>i$, we have $\Delta(j) \geqslant 0$. Otherwise, we would have $|v[i+1, j]|_{1}-$ $|u[i+1, j]|_{1}>1$ contradicting the fact that $y$ is balanced. Similarly, for all $j<i$, we also have $\Delta(j) \geqslant 0$.

Since $u$ and $v$ are distinct, replacing $u$ with $v$ if needed, we may assume that there exists a minimal $i \in\{0, \ldots, n-1\}$ such that $\Delta(i)=1$. From the above discussion and the minimality of $i, \Delta(j)=0$ for $j<i$ and $\Delta(j) \in\{0,1\}$ for $j>i$.

From (3), for any $j<n$, we have

$$
\begin{aligned}
& \Delta(j+1)>\Delta(j) \Rightarrow S(u, j+1)-S(v, j+1)=S(u, j)-S(v, j)+(n-j) \\
& \Delta(j+1)=\Delta(j) \Rightarrow S(u, j+1)-S(v, j+1)=S(u, j)-S(v, j) \\
& \Delta(j+1)<\Delta(j) \Rightarrow S(u, j+1)-S(v, j+1)=S(u, j)-S(v, j)-(n-j) .
\end{aligned}
$$

In view of these observations, the knowledge of $\Delta(0), \Delta(1), \ldots$ permits to compute $(S(u, j)-S(v, j))_{0 \leqslant j<n}$ and we deduce that $0<S(u)-S(v)<n+1$ concluding the proof.

Proof (Proof of Theorem 2). Let $x$ be a Sturmian word of type 0 and $m \geqslant 2$. From (1), we have, for all $\ell \geqslant 0$,

$$
\mathbf{b}_{x}^{(2)}(\ell) \leqslant \mathbf{b}_{x}^{(m)}(\ell) \leqslant p_{x}(\ell)=\ell+1 .
$$

We just need to show that any two distinct factors of length $\ell$ in $x$ are not 2-binomially equivalent, i.e., $\ell+1 \leqslant \mathbf{b}_{x}^{(2)}(\ell)$.

Proceed by contradiction. Assume that $x$ contains two distinct factors $u$ and $v$ that are 2-binomially equivalent. In particular, $\left(\begin{array}{c}u \\ 00\end{array}\right)=\left(\begin{array}{c}v \\ 00\end{array}\right)$ and $\left(\begin{array}{c}u \\ 11\end{array}\right)=\left(\begin{array}{c}v \\ 11\end{array}\right)$. Hence we get $|u|=|v|$ and $|u|_{1}=|v|_{1}=n$. From Corollary 1, there exist $k \geqslant 1$ and a Sturmian word $y$ such that

$$
u=0^{r} 10^{k+\epsilon_{0}} 10^{k+\epsilon_{1}} 1 \cdots 0^{k+\epsilon_{n-1}} 10^{s}, \quad v=0^{r^{\prime}} 10^{k+\epsilon_{0}^{\prime}} 10^{k+\epsilon_{1}^{\prime}} 1 \cdots 0^{k+\epsilon_{n-1}^{\prime}} 10^{s^{\prime}}
$$

where $\epsilon=\epsilon_{0} \epsilon_{1} \cdots \epsilon_{n-1}$ and $\epsilon^{\prime}=\epsilon_{0}^{\prime} \epsilon_{1}^{\prime} \cdots \epsilon_{n-1}^{\prime}$ are both factors of $y$.

Since $u \sim_{2} v$, it follows $\left(\begin{array}{c}u \\ 01\end{array}\right)=\left(\begin{array}{c}v \\ 01\end{array}\right)$. From Remark 4 , we get

$$
r(n+1)+S(\epsilon)+k \frac{n(n+1)}{2}=r^{\prime}(n+1)+S\left(\epsilon^{\prime}\right)+k \frac{n(n+1)}{2} .
$$

Otherwise stated, we get $S(\epsilon)-S\left(\epsilon^{\prime}\right)=\left(r^{\prime}-r\right)(n+1)$ contradicting the previous lemma. 


\section{The Case of the Thue-Morse Word}

The Thue-Morse word $t=01101001100101101001011001101001 \cdots$ is the infinite word $\lim _{n \rightarrow \infty} \varphi^{n}(a)$ where $\varphi: 0 \mapsto 01,1 \mapsto 10$. The factor complexity of the Thue-Morse word is well-known $[2,5]: p_{t}(0)=1, p_{t}(1)=2, p_{t}(2)=4$ and

$$
p_{t}(n)=\left\{\begin{array}{l}
4 n-2 \cdot 2^{m}-4 \text { if } 2 \cdot 2^{m}<n \leqslant 3 \cdot 2^{m} \\
2 n+4 \cdot 2^{m}-2 \text { if } 3 \cdot 2^{m}<n \leqslant 4 \cdot 2^{m}
\end{array}\right.
$$

and the abelian complexity of $t$ is obvious.

Lemma 5. We have $\mathbf{b}_{t}^{(1)}(2 n)=3$ and $\mathbf{b}_{t}^{(1)}(2 n+1)=2$ for all $n \geqslant 1$.

The main result of this section is the following one. It is quite in contrast with the Sturmian case because here, the Thue-Morse word exhibits a bounded $m$-binomial complexity.

Theorem 4. Let $m \geqslant 2$. There exists $C_{m}>0$ such that the $m$-binomial complexity of the Thue-Morse word satisfies $\mathbf{b}_{t}^{(m)}(n) \leqslant C_{m}$ for all $n \geqslant 0$.

For the sake of presentation, we first show that the 2-binomial complexity of the Thue-Morse word is bounded by a constant.

Theorem 5. There exists $C_{2}>0$ such that the 2-binomial complexity of the Thue-Morse word satisfies $\mathbf{b}_{t}^{(2)}(n) \leqslant C_{2}$ for all $n \geqslant 0$.

Proof. Any factor $v$ of $t$ admits a factorization of the kind $p \varphi(u) s$ with $p, s \in$ $\{0,1, \varepsilon\}$ and where $u$ is a factor of $t$. Using Remark 2, it is therefore enough to prove that, for all $n$,

$$
\#\left\{\mathbf{B}^{(2)}(v) \mid \exists u \in \operatorname{Fac}_{t}(n): v=\varphi(u)\right\} \leqslant 9 .
$$

Recall from the proof of Lemma 3 that the 2-binomial equivalence class of a word $v$ of length $2 n$ over a binary alphabet $\{0,1\}$ is completely determined by its length, $|v|_{0}$ and $\left(\begin{array}{c}v \\ 01\end{array}\right)$, i.e.,

$$
\begin{aligned}
& \#\left\{\mathbf{B}^{(2)}(v) \mid \exists u \in \mathrm{Fac}_{t}(n): v=\varphi(u)\right\} \\
= & \#\left\{\left(\left(\begin{array}{c}
v \\
0
\end{array}\right),\left(\begin{array}{c}
v \\
1
\end{array}\right),\left(\begin{array}{c}
v \\
00
\end{array}\right),\left(\begin{array}{c}
v \\
01
\end{array}\right),\left(\begin{array}{c}
v \\
10
\end{array}\right),\left(\begin{array}{c}
v \\
11
\end{array}\right)\right) \mid \exists u \in \mathrm{Fac}_{t}(n): v=\varphi(u)\right\} \\
= & \#\left\{\left(|v|_{0},\left(\begin{array}{c}
v \\
01
\end{array}\right)\right) \mid \exists u \in \operatorname{Fac}_{t}(n): v=\varphi(u)\right\} .
\end{aligned}
$$

Fix $n \geqslant 1$. Consider an arbitrary factor $u=u_{0} \cdots u_{n-1} \in \operatorname{Fac}_{t}(n)$ and the corresponding factor $v=\varphi(u)=v_{0} \cdots v_{2 n-1}$ of $t$ of length $2 n$. From Lemma 5 , $|v|_{0}$ takes at most three values (depending on $n$ ).

Let us compute the possible values taken by the coefficient $\left(\begin{array}{c}v \\ 01\end{array}\right)$. Consider an occurrence of 01 as a subword of $v$, i.e., a pair $(i, j), i<j \leqslant n-1$, such that $v_{i} v_{j}=01$. There are two possible cases: 
- If $i=2 m$ and $j=2 m+1$, for some $m \geqslant 0$, then $u_{m}=0$ because $v_{2 m} v_{2 m+1}=$ $\varphi\left(u_{m}\right)$. There are $|u|_{0}$ such occurrences.

- Otherwise, we have $i \in\{2 m, 2 m+1\}, j \in\left\{2 m^{\prime}, 2 m^{\prime}+1\right\}$ with $m^{\prime}>m$. For all $m$ (resp. $m^{\prime}$ ), exactly one letter of the factor $v_{2 m} v_{2 m+1}=\varphi\left(u_{m}\right)$ (resp. $v_{2 m^{\prime}} v_{2 m^{\prime}+1}=\varphi\left(u_{m}^{\prime}\right)$ ) is 0 and the other one is 1. Hence, for any $i \in\{0, \ldots, n-2\}, j$ can take a value of the $n-1-i$ values in $\{i+1, \ldots, n-1\}$.

Summarizing these two cases, we have

$$
\left(\begin{array}{c}
v \\
01
\end{array}\right)=|u|_{0}+\sum_{i=0}^{n-2}(n-1-i)=|u|_{0}+\frac{n(n-1)}{2} .
$$

From Lemma $5,|u|_{0}$ takes at most three values (depending on $n$ ) and therefore the same holds for $\left(\begin{array}{c}v \\ 01\end{array}\right)$. Hence, the conclusion follows.

We now extend the proof of Theorem 5. The first part is to generalize (4).

Lemma 6. Let $m, k \geqslant 1$. Assume that there exists $D$ such that, for all $n$,

$$
\#\left\{\mathbf{B}^{(m)}(v) \mid \exists u \in \operatorname{Fac}_{t}(n): v=\varphi^{k}(u)\right\} \leqslant D .
$$

Then the m-binomial complexity of the Thue-Morse word $\mathbf{b}_{t}^{(m)}$ is bounded by a constant.

Proof. Let $\ell \geqslant 1$. Let $f$ be a factor of $t$ of length $\ell$. This factor is of the form ${ }^{3}$ pvs where $p$ (resp. $s$ ) is a proper suffix (resp. prefix) of some $\varphi^{k}(a)\left(\operatorname{resp} . \varphi^{k}(b)\right)$ where $a, b$ are letters and $v=\varphi^{k}(u)$ for some factor $u$ of $t$ of length $n$. In particular, we have $|p|,|q| \leqslant 2^{k}-1$. Note that $\ell$ is of the form $n \cdot 2^{k}+r$ with $0 \leqslant r \leqslant 2\left(2^{k}-1\right)$. Hence, for a given $f$ of length $\ell$, the corresponding integer $n$ can take at most 2 values which are $\left\lfloor\ell / 2^{k}\right\rfloor-1$ and $\left\lfloor\ell / 2^{k}\right\rfloor$. From the assumption, we get

$$
\#\left\{\mathbf{B}^{(m)}(v) \mid \exists u \in \operatorname{Fac}_{t}\left(\left\lfloor\ell / 2^{k}\right\rfloor-1\right) \cup \operatorname{Fac}_{t}\left(\left\lfloor\ell / 2^{k}\right\rfloor\right): v=\varphi^{k}(u)\right\} \leqslant 2 D .
$$

Finally, using Remark 2, we have $\mathbf{B}^{(m)}(f)=\mathbf{B}^{(m)}(p) \circ \mathbf{B}^{(m)}(v) \circ \mathbf{B}^{(m)}(s)$. Since $p$ and $s$ have bounded length, $\mathbf{B}^{(m)}(p)$ and $\mathbf{B}^{(m)}(s)$ take a bounded number of values. Moreover, $\mathbf{B}^{(m)}(v)$ takes at most $2 D$ values, hence $\mathbf{b}_{t}^{(m)}$ is bounded by constant.

From now on, intervals $[r, s]$ (resp. $[r, s)$ ) will be considered as intervals of integers, i.e., one should understand $[r, s] \cap \mathbb{Z}($ resp. $[r, s) \cap \mathbb{Z})$.

Aside from the idea of dealing with words of a convenient form, the second key idea of the proof of Theorem 5 is to split the set of occurrences of the subword 01 into two disjoint subsets facilitating the counting. We shall now generalize this idea for $m$-binomial complexity but some terminology is required. Let $v$ be a word. A subset $T=\left\{t_{1}<t_{2}<\ldots<t_{n}\right\} \subseteq[0,|v|)$ defines a subword denoted by $v_{T}=v_{t_{1}} v_{t_{2}} \cdots v_{t_{n}}$.

\footnotetext{
${ }^{3}$ This is the idea of "de-substitution" where $t$ is factorized into consecutive factors of length $2^{k}$.
} 
Definition 3. If $\alpha_{1}, \ldots, \alpha_{m}$ are non-empty and pairwise disjoint subsets of a set $X$ such that $\cup_{i} \alpha_{i}=X$, then $\alpha=\left\{\alpha_{1}, \ldots, \alpha_{m}\right\}$ is a partition of $X$. Any partition $\alpha$ of a set $X$ is a refinement of a partition $\beta$ of $X$ if every element of $\alpha$ is a subset of some element of $\beta$. In that case, $\alpha$ is said to be finer than $\beta$ (equivalently $\beta$ is coarser than $\alpha$ ) and we write $\alpha \preceq \beta$. Since $\preceq$ is a partial order, we define a chain as a subset of partitions $\beta^{(1)}, \beta^{(2)}, \ldots$ of $X$ satisfying

$$
\beta^{(1)} \preceq \beta^{(2)} \preceq \cdots .
$$

$A$-partition $\alpha=\left\{\alpha_{1}, \ldots, \alpha_{m}\right\}$ of the set $[0, m k)$ is a partition into subsets $\alpha_{i}=[(i-1) k, i k)$ of size $k$. In particular, a $2^{i}$-partition is a refinement of a $2^{j}$-partition of $\left[0,2^{k}\right), i<j \leqslant k$.

Definition 4. Let $X$ be a set and $T=\left\{t_{1}<t_{2}<\ldots<t_{n}\right\}$ be a subset of $X$. A partition $\alpha=\left\{\alpha_{1}, \ldots, \alpha_{m}\right\}$ of $X$ induces a partition $\alpha_{T}=\left\{\gamma_{1}, \ldots, \gamma_{r}\right\}$ of $[1, n]$ defined by

$$
i, j \in \gamma_{t} \Leftrightarrow \exists s: t_{i}, t_{j} \in \alpha_{s} .
$$

Note that for two partitions $\alpha, \beta$ of $X$, if $\alpha \preceq \beta$, then $\alpha_{T} \preceq \beta_{T}$.

Example 2. Take $X=[0,7]$ and $T=\{0,2,3,5\}$. Consider the following two partitions of $X: \alpha=\{\{0,1\},\{2,3,4\},\{5,6,7\}\}$ and $\beta=\{\{0,1,2\},\{3,4,5\},\{6,7\}\}$. We get $\alpha_{T}=\{\{1\},\{2,3\},\{4\}\}$ and $\beta_{T}=\{\{1,2\},\{3,4\}\}$.

Definition 5. Let $T=\left\{t_{1}<t_{2}<\ldots<t_{n}\right\}$ and $U=\left\{u_{1}<u_{2}<\ldots<u_{n}\right\}$ be subsets of $X$. These subsets are equidistributed with respect to a partition $\alpha$ of $X$ if $\alpha_{T}=\alpha_{U}$. These subsets are equidistributed with respect to a chain $\mathfrak{C}$ of partitions of $X$ if $\alpha_{T}=\alpha_{U}$ for all $\alpha \in \mathfrak{C}$. We also say that the subsets are $\mathfrak{C}$-equidistributed.

Example 3. Consider the chain $\mathfrak{C}$ consisting of the 4-partition $\beta=\{[0,3],[4,7]\}$ and the 2-partition $\alpha=\{[0,1],[2,3],[4,5],[6,7]\}$ of the set $[0,7]$. The subsets $T=\{0,5\}, U=\{1,2\}$ and $V=\{3,4\}$ are equidistributed with respect to the 2-partition $\left(\alpha_{T}=\alpha_{U}=\alpha_{V}=\{\{1\},\{2\}\}\right)$, but $U$ is not $\mathfrak{C}$-equidistributed to $T$ (resp. $V$ ) because $\beta_{T}=\beta_{V}=\{\{1\},\{2\}\}$ and $\beta_{U}=\{\{1,2\}\}$.

Example 4. In the last part of the proof of Theorem 5, we have considered the two possible cases for an occurrence of the subword 01 in $v$. If $T=\{i, j\}$ is a subset of $[0,|v|)$ and $\alpha$ is the 2-partition of $[0,|v|)$, then these cases correspond exactly to the two possible values $\alpha_{T}=\{1,2\}$ or $\alpha_{T}=\{\{1\},\{2\}\}$.

Let $\mathfrak{C}$ be a chain $\beta^{(1)} \preceq \beta^{(2)} \preceq \cdots$ of partitions of $X$ and $T=\left\{t_{1}, \ldots, t_{n}\right\}$ be a subset of $X$. We use nested brackets to represent the induced chain $\beta_{T}^{(1)} \preceq \beta_{T}^{(2)} \preceq$ $\cdots$ of partitions of $[1, n]$. The outer (resp. inner) brackets represent the coarsest (resp. finest) partition of $[1, n]$. As an example $\left[\left[t_{1} t_{2}\right]\right]\left[\left[t_{3}\right]\left[t_{4}\right]\right]$ represents the partition $\{\{1,2\},\{3\},\{4\}\}$ and the coarser partition $\{\{1,2\},\{3,4\}\}$. To get used to these new definitions, we consider another particular statement. (A precise and formal definition of the bracket notation is given in [15].) 
Remark 5. Two subsets $T$ and $U$ of size $n$ of $X$ are equidistributed with respect to a chain $\mathfrak{C}$ of partitions of $X$ if and only if they give rise to the same notation of nested brackets. We call it the type of $T$ with respect to $\mathfrak{C}$.

Example 5 (continuing Example 3). Consider the subsets $R=\{0,1,4,7\}$ and $S=\{2,3,4,6\}$ of $[0,7]$. We have $\alpha_{R}=\alpha_{S}=\{\{1,2\},\{3\},\{4\}\}$ and $\beta_{R}=\beta_{S}=$ $\{\{1,2\},\{3,4\}\}$. Hence $R$ and $S$ are $\mathfrak{C}$-equidistributed and give both rise to the notation $\left[\left[t_{1} t_{2}\right]\right]\left[\left[t_{3}\right]\left[t_{4}\right]\right]$.

We prove the case of the 3-binomial complexity. The proof of the general case has been treated in [15].

Theorem 6. There exists $C_{3}>0$ such that the 3-binomial complexity of the Thue-Morse word satisfies $\mathbf{b}_{t}^{(3)}(n) \leqslant C_{3}$ for all $n \geqslant 0$.

Proof. In view of Lemma 6 , it is enough to show that there exists a constant $D$ such that, for all $n$, we have $\#\left\{\mathbf{B}^{(3)}(v) \mid \exists u \in \operatorname{Fac}_{t}(n): v=\varphi^{2}(u)\right\} \leqslant D$.

Let $n \geqslant 1$. Let $v=\varphi^{2}(u)$ with $u \in \operatorname{Fac}_{t}(n)$. In particular, $|v|=4 n$. Consider the chain $\mathfrak{C}$ consisting of the 2 -partition and the 4 -partition of $[0,4 n)$. Any subset $T=\left\{t_{1}<t_{2}<t_{3}\right\}$ of $[0,4 n)$ is $\mathfrak{C}$-equidistributed to a subset of one the following types:

$-\left[t_{1}\right]\left[t_{2}\right]\left[t_{3}\right]$, i.e., the union of the types $\left[\left[t_{1}\right]\right]\left[\left[t_{2}\right]\right]\left[\left[t_{3}\right]\right],\left[\left[t_{1}\right]\left[t_{2}\right]\right]\left[\left[t_{3}\right]\right]$ and $\left[\left[t_{1}\right]\right]\left[\left[t_{2}\right]\left[t_{3}\right]\right]$ : the 3 elements of $T$ belong to pairwise distinct subsets of the 2 -partition of $[0,4 n)$

- $\left[\left[t_{1} t_{2}\right]\left[t_{3}\right]\right]$ or $\left[\left[t_{1}\right]\left[t_{2} t_{3}\right]\right]$ : two elements belong to the same subset of the 2partition of $[0,4 n)$ and the 3 elements of $T$ belong to the same subset of the 4-partition of $[0,4 n)$.

- $\left[\left[t_{1} t_{2}\right]\right]\left[\left[t_{3}\right]\right]$ or $\left[\left[t_{1}\right]\right]\left[\left[t_{2} t_{3}\right]\right]$ : two elements belong to the same subset of the 2 -partition and to the same subset of the 4-partition of $[0,4 n)$.

Let $e=e_{0} e_{1} e_{2}$ be a word of length 3 . We will count the number of occurrences of the subword $e=v_{t_{1}} v_{t_{2}} v_{t_{3}}$ in $v$ depending on the type of $T=\left\{t_{1}, t_{2}, t_{3}\right\}$ with respect to $\mathfrak{C}$.

Assume that the type of $T$ is $\left[t_{1}\right]\left[t_{2}\right]\left[t_{3}\right]$. Each subset $S$ of the 2-partition of $[0,4 n)$ corresponds to a factor $v_{S}=01$ or $v_{S}=10$ and $v$ contains $2 n$ such factors. Hence the number of subwords $e$ occurring in $v$ for this type takes, for a given $n$, a unique value which is $\left(\begin{array}{c}2 n \\ 3\end{array}\right)$.

Now assume that the type of $T$ is $\left[\left[t_{1} t_{2}\right]\left[t_{3}\right]\right]$ (similar arguments apply to $\left.\left[\left[t_{1}\right]\left[t_{2} t_{3}\right]\right]\right)$. Each subset $S$ of the 4-partition of $[0,4 n)$ corresponds to a factor $v_{S}$ which is either $\varphi^{2}(0)=0110$ or $\varphi^{2}(1)=1001$. Then the number of subwords $e$ occurring in $v$ of this type is

$$
\underbrace{\left(\begin{array}{c}
01 \\
e_{0} e_{1}
\end{array}\right)}_{0 \text { or } 1} \underbrace{\left(\begin{array}{c}
10 \\
e_{2}
\end{array}\right)}_{1}|u|_{0}+\underbrace{\left(\begin{array}{c}
10 \\
e_{0} e_{1}
\end{array}\right)}_{0 \text { or } 1} \underbrace{\left(\begin{array}{c}
01 \\
e_{2}
\end{array}\right)}_{1}|u|_{1} \in\left\{0,|u|_{0},|u|_{1}\right\} .
$$


Recall that, for a given $n=|u|$, the pair $\left(|u|_{0},|u|_{1}\right)$ can take at most three values (see Lemma 5). The number of subwords $e$ occurring in $v$ of this type takes, for a given $n$, takes at most 4 values ${ }^{4}$.

Now assume that the type of $T$ is $\left[\left[t_{1} t_{2}\right]\right]\left[\left[t_{3}\right]\right]$ (similar arguments apply to $\left.\left[\left[t_{1}\right]\right]\left[\left[t_{2} t_{3}\right]\right]\right)$. Each subset $S$ of the 4 -partition of $[0,4 n)$ is a union of two sets $S^{\prime}, S^{\prime \prime}$ of the 2-partition of $[0,4 n)$ and we have either $v_{S^{\prime}}=01, v_{S^{\prime \prime}}=10$ or $v_{S^{\prime}}=10, v_{S^{\prime \prime}}=01$. They are $n$ subsets of size 4 in the 4 -partition of $[0,4 n)$ and we have to pick 2 of them. Hence, the number of subwords $e$ occurring in $v$ for this type is

$$
(\underbrace{\left(\begin{array}{c}
01 \\
e_{0} e_{1}
\end{array}\right)+\left(\begin{array}{c}
10 \\
e_{0} e_{1}
\end{array}\right)}_{0 \text { or } 1})(\underbrace{\left(\begin{array}{c}
01 \\
e_{2}
\end{array}\right)+\left(\begin{array}{c}
10 \\
e_{2}
\end{array}\right)}_{2})\left(\begin{array}{l}
n \\
2
\end{array}\right)
$$

and this quantity, for a given $n$, takes at most 2 values.

We have proved that, for all $|e|=3$ and $v=\varphi^{2}(u)$ with $u \in \operatorname{Fac}_{t}(n),\left(\begin{array}{l}v \\ e\end{array}\right)$ takes at most $1+2 \cdot 4+2 \cdot 2=13$ values (these values depend on $n$, but the number of values is bounded without any dependence to $n)$. Note that $\mathbf{B}^{(3)}(v)$ is determined from $\mathbf{B}^{(2)}(v)$ and by the values of $\left(\begin{array}{c}v \\ e\end{array}\right)$ for the words $e$ of length 3 . To conclude the proof, note that $\#\left\{\mathbf{B}^{(2)}(v) \mid \exists u \in \mathrm{Fac}_{t}(n): v=\varphi^{2}(u)\right\}$ is bounded by $\#\left\{\mathbf{B}^{(2)}(v) \mid \exists z \in \operatorname{Fac}_{t}(2 n): v=\varphi(z)\right\} \leqslant 9$ using (4). Consequently, we have

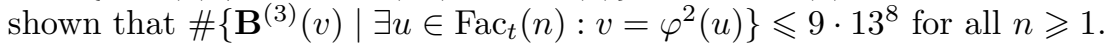

Remark 6. By computer experiments, $\mathbf{b}_{t}^{(2)}(n)$ is equal to 9 if $n \equiv 0(\bmod 4)$ and to 8 otherwise, for $10 \leqslant n \leqslant 1000$. Moreover, $\mathbf{b}_{t}^{(3)}(n)$ is equal to 21 if $n \equiv 0$ $(\bmod 8)$ and to 20 otherwise, for $8 \leqslant n \leqslant 500$.

\section{A Glimpse at Avoidance}

It is obvious that, over a 2-letter alphabet, any word of length $\geqslant 4$ contains a square. On the other hand, there exist square-free infinite ternary words [12]. In the same way, over a 3-letter alphabet, any word of length $\geqslant 8$ contains an abelian square, i.e., a word $u u^{\prime}$ where $u \sim_{1} u^{\prime}$. But, over a 4-letter alphabet, abelian squares are avoidable, see for instance [10]. So a first natural question in that direction is to determine, whether or not, over a 3-letter alphabet 2-binomial squares can be avoided in arbitrarily long words. Naturally, a 2-binomial square is a word of the form $u u^{\prime}$ where $u \sim_{2} u^{\prime}$. Note that, for abelian equivalence, the longest ternary word which is 2-abelian square-free has length 537 [9].

As an example, $u=121321231213123132123121312$ is a word of length 27 without 2-binomial squares but this word cannot be extended without getting a 2-binomial square. Indeed, $u 1$ (resp. $u 3$ ) ends with a square of length 8 (resp. 26)

Consider the 13-uniform morphism of Leech [11] which is well-known to be square-free, $g: a \mapsto a b c b a c b c a b c b a, b \mapsto b c a c b a c a b c a c b, c \mapsto c a b a c b a b c a b a c$. In

\footnotetext{
${ }^{4}$ A close inspection shows that if $|u|=2 n$, then $|u|_{0},|u|_{1} \in\{n-1, n, n+1\}$, if $|u|=2 n+1$, then $|u|_{0},|u|_{1} \in\{n, n+1\}$.
} 
the submitted version of this paper, we conjectured that the infinite square-free word $g^{\omega}(1)$ avoids 2-binomial squares. For instance, we can prove that

$$
u \sim_{2} v \Leftrightarrow g(u) \sim_{2} g(v) .
$$

Nevertheless, M. Bennett has recently shown that the factor of length 508 occurring in position 845 is a 2-binomial square [4].

\section{Acknowledgments}

The idea of this binomial equivalence came after the meeting "Representing streams" organized at the Lorentz center in December 2012 where Jean-Eric Pin presented a talk, Noncommutative extension of Mahlers theorem on interpolation series, involving binomial coefficients on words. Jean-Eric Pin and the first author proposed independently to introduce this new relation.

\section{References}

1. S.V. Avgustinovich, D.G. Fon-Der-Flaass, A.E. Frid, Arithmetical complexity of infinite words, in Words, Languages \& Combinatorics III, M. Ito and T. Imaoka (Eds.), World Scientific Publishing (2003) 51-62.

2. S. Brlek, Enumeration of factors in the Thue-Morse word, Discrete Appl. Math. 24 (1989), 83-96.

3. J. Berstel, M. Crochemore, J.-E. Pin, Thue-Morse sequence and $p$-adic topology for the free monoid, Disc. Math. 76 (1989), 89-94.

4. J. Currie, personal communication, 3th June 2013.

5. A. de Luca, S. Varricchio, On the factors of the Thue-Morse word on three symbols, Inform. Process. Lett. 27 (1988), 281-285.

6. P. Erdős, Some unsolved problems, Magyar Tud. Akad. Mat. Kutató Int. Közl. 6 (1961), 221-254.

7. T. Kamae, L. Zamboni, Sequence entropy and the maximal pattern complexity of infinite words, Ergodic Theory Dynam. Systems 22 (2002), 1191-1199.

8. J. Karhumäki, A. Saarela, L. Q. Zamboni, On a generalization of Abelian equivalence and complexity of infinite words, arXiv:1301.5104.

9. M. Huova, J. Karhumäki, Observations and problems on $k$-abelian avoidability, In Combinatorial and Algorithmic Aspects of Sequence Processing (Dagstuhl Seminar 11081), (2011) 2215-2219.

10. V. Keränen, Abelian squares are avoidable on 4 letters, Lecture Notes in Comput. Sci. 623 (1992), 41-52.

11. J. Leech, A problem on strings of beads, Math. Gazette 41, 277-278 (1957).

12. M. Lothaire, Combinatorics on Words, Cambridge Mathematical Library, Cambridge University Press, (1997).

13. M. Lothaire, Algebraic Combinatorics on Words, Encyclopedia of Mathematics and its Applications 90, Cambridge University Press (2002).

14. N. Pytheas Fogg, Substitutions in dynamics, arithmetics and combinatorics, V. Berthé, S. Ferenczi, C. Mauduit and A. Siegel (Eds.), Lecture Notes in Mathematics 1794, Springer-Verlag, Berlin, 2002.

15. M. Rigo, P. Salimov, Another Generalization of Abelian Equivalence: Binomial Complexity of Infinite Words (long version), preprint (2013), available at http://hdl.handle.net/2268/149313 